\title{
Small Talk mit Bildzeichen
}

Der Beitrag von Emojis zur digitalen Alltagskommunikation

\author{
Michael Beißwenger · Steffen Pappert
}

Online publiziert: 29. Januar 2020

(C) Der/die Autor(en) 2020

Zusammenfassung Emojis erfreuen sich in der digitalen Alltagskommunikation großer Beliebtheit. Insbesondere in informellen, privaten Kontexten werden die kleinen Bildzeichen hochfrequent verwendet. Dies lässt annehmen, dass sie die Interaktionsgestaltung und das sprachliche Handeln sehr zentral und differenziert unterstützen. Im Beitrag wird ein Beschreibungsrahmen vorgestellt, der Praktiken des Handelns mit Emojis unter einer pragmatischen Perspektive analysierbar macht und der danach fragt, was Emojis für den Aufbau von Sinn und Verstehen in der internetbasierten Kommunikation leisten (und warum). Wie dieser Beschreibungsrahmen für das Verständnis konkreter Emoji-Verwendungen im Interaktionskontext genutzt werden kann, wird an der Analyse eines WhatsApp-Verlaufs demonstriert.

Schlüsselwörter Pragmatik - Semiotik · Interaktionsgestaltung - Internetbasierte Kommunikation $\cdot$ Emoji $\cdot$ WhatsApp

M. Beißwenger ( $\bowtie) \cdot$ S. Pappert

Institut für Germanistik, Universität Duisburg-Essen, Essen, Deutschland

E-Mail: michael.beisswenger@uni-due.de

S. Pappert

E-Mail: steffen.pappert@uni-due.de 


\title{
Small Talk with Emojis
}

How Pictograms Contribute to the Organisation of Computer-Mediated Conversation

\begin{abstract}
Emojis have emerged as one of the most salient features of computermediated communication (CMC) in recent years. Under a pragmatic perspective the frequent use of emoji icons especially in private, informal instances of CMC (e.g. in whatsapp conversations) suggests that they make an important contribution to the organisation of understanding and social relations. In our article we present a framework for the pragmatic analysis of emoji occurences in CMC data and demonstrate the use of this framework on the example of the emoji practices found in a private whatsapp interaction.
\end{abstract}

Keywords Pragmatics · Semiotics · Interaction · Computer-Mediated Communication $\cdot$ Emoji $\cdot$ Whatsapp

\section{Einleitung}

»Mehr als $90 \%$ derer, die im Netz kommunizieren, nutzen Emojis und Emoticons. Doch was wollen und können sie uns damit sagen?« (Anderl 2017). Solche und vergleichbare Aufhänger findet man allenthalben - in (Online-)Zeitungen und Zeitschriften, vom SPIEGEL bis zur Bild der Frau. Emojis sind demzufolge in aller Munde - oder besser: unter jedermanns/-fraus Fingern und Augen. Die große Beliebtheit, derer sich Emojis in bestimmten Formen der digitalen Alltagskommunikation erfreuen, lässt annehmen, dass es sich bei ihnen nicht nur um unverbindliches Beiwerk des digital vermittelten sozialen Kontakts handelt, sondern um Einheiten, die für ihre Verwenderinnen und Verwender wichtige Funktionen übernehmen. Unter einer pragmatischen Perspektive ist nichts, was Menschen in Kommunikation tun, verzichtbar. Wie wir kommunikative Äußerungen gestalten und welche Mittel wir dafür nutzen, lässt sich aus Zwecken des Handelns begründen und aus unserer Einschätzung, was unter den gegebenen situativen Bedingungen notwendig und erforderlich ist, um diese Zwecke bestmöglich, sozialverträglich und auf angemessene Art und Weise zu realisieren.

Dem in der massenmedialen Berichterstattung gespiegelten öffentlichen Interesse an und dem vielfältigen Gebrauch von Emojis in der internetbasierten Kommunikation stehen bislang nur wenige linguistische Untersuchungen gegenüber, die sich mit dem Gebrauch der kleinen Bildzeichen auseinandersetzen (vgl. z. B. Kelly/Watts 2015; Cramer et al. 2016; Danesi 2017; Dürscheid/Siever 2017; Herring/ Dainas 2017; Pappert 2017; Siebenhaar 2018; Dürscheid/Meletis 2019; Beißwenger/ Pappert 2019a, 2019b, 2019c; Imo/Fladrich 2020; für einen ausführlichen Überblick zum Stand der Forschung s. Beißwenger/Pappert 2019a, S. 11-27). Gleichwohl wird von den verschiedenen Autorinnen und Autoren das angesprochene Desiderat durchaus erkannt und benannt. So betont Imo (2019, S. 51), dass bei der Forschung zur multimodalen Interaktion über Kurznachrichten u.a. die »Bandbreite an Funktionen« von Emojis in den Blick zu nehmen sei. Dürscheid (2016, S. 459) plädiert vor dem Hintergrund der unterschiedlichen semiotischen Ressourcen in digitaler 


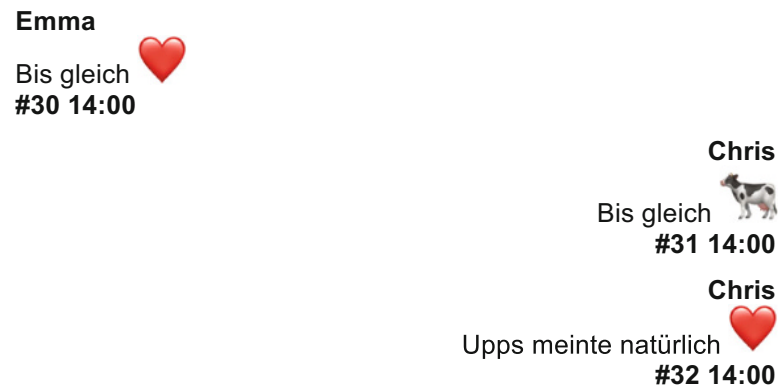

\title{
Emma
}

Du

\#33 16:01

Emma

\#34 16:01

\begin{abstract}
Abb. 1 https://db.mocoda2.de/\#/view/na2Ua (Bei dem Beispiel handelt es sich um einen Ausschnitt aus einem längeren Chatverlauf. Dieser kann unter der angegebenen URL im Online-Korpus Mobile Communication Database 2 (MoCoDa2, vgl. Beißwenger et al. 2019) nachvollzogen werden)
\end{abstract}

Schriftlichkeit dafür, diese nicht nur in ihrer Systematik zu beschreiben, »sondern auch hinsichtlich ihrer pragmatischen Funktion genauer zu betrachten «.

Dass Emojis fraglos wichtige Beiträge zur Herstellung von Sinn und sozialem Verstehen leisten, lässt sich an Interaktionsausschnitten wie dem in Beispiel 1 wiedergegebenen zeigen, in dem es Chris offenbar wichtig ist, ein (vermutlich ver-

sehentlich) verwendetes Emoji ( 5 is) nachträglich metakommunikativ zu korrigieren, d.h. durch die eigentlich intendierte Form $\mathrm{zu}$ ersetzen. Es macht für die Kommunizierenden einen Unterschied, welche Emoji-Form an einer bestimmten Stelle eines Beitrags oder einer Beitragssequenz steht. Stellt man sich den Ausschnitt in Abb. 1 ganz ohne Emojis vor, so ergäbe sich hinsichtlich der Postings \#30 und \#31 (angenommen, in \#31 stünde das von Chris eigentlich intendierte Herz) auf der propositionalen und auf der Handlungsebene kein Unterschied, wohl aber auf der sozialen: Die beiden Kommunizierenden verwenden Herz-Emojis, um ihre Beziehung ins Bild zu setzen und sich damit - obwohl vermutlich an dieser Stelle des Austauschs unstrittig - wechselseitig und nichtsprachlich ihrer ungebrochenen, wechselseitigen Wertschätzung bzw. Zuneigung zu versichern. Die Postings \#33 und \#34 hingegen wären ohne Emojis nicht unmittelbar interpretierbar: In \#33 realisiert das Emoji ein notwendiges Element des syntaktischen Strukturaufbaus und steht anstelle eines Wortes; das Posting wäre nach Abzug des Bildelements propositional nicht erschließbar und für Emma vermutlich auch kaum als Handlung konstituierbar. Posting \#34 kommt gänzlich ohne sprachliche Einheiten aus; gehandelt wird hier ausschließlich mit Bildzeichen. Posting \#32 ist ein Sonderfall, da aufgrund des me- 
takommunikativen Akts hier eine erwähnte Verwendung einer Emoji-Form vorliegt mit dem Zweck, die Emoji-Verwendung aus \#31 zu reparieren und der Intention, Emma dazu zu veranlassen, die in \#31 wahrgenommene Verwendung des Kuh-Emojis in ihrem mentalen Kommunikationsprotokoll durch eine Instanz des Herz-Emojis zu überschreiben.

In diesem Beitrag möchten wir Emojis hinsichtlich ihrer pragmatischen Funktionen betrachten: Wir interessieren uns dafür, was Emojis in und für die Organisation zwischenmenschlicher Kommunikation leisten und in welchen Funktionen Emojis von Kommunikationsbeteiligten, die zueinander in bestimmten Beziehungen stehen, auf dem Hintergrund konkreter Kommunikationsanlässe und einer jeweiligen kommunikativen Vorgeschichte genutzt werden. Da unterschiedliche Kommunikationsanwendungen und -plattformen ganz unterschiedliche Rahmenbedingungen für die Gestaltung von Kommunikation bereitstellen, konzentrieren wir uns auf die EmojiVerwendung in WhatsApp-Chats. WhatsApp ist der zurzeit populärste Messengerdienst, der von mehr als 1,5 Mrd. Menschen weltweit genutzt wird. ${ }^{1}$ Im Gegensatz zur internetbasierten Kommunikation in Foren, Blogs, Twitter, Facebook oder Instagram stellt WhatsApp »eine private, nicht-öffentliche Kommunikationsform « (Imo 2017, S. 97) dar und ist deshalb als Anwendung für die Realisierung von Alltagskommunikation besonders beliebt. Entsprechend finden sich in WhatsApp zahlreiche Beispiele für Small Talk, also für Formen der privaten Alltagskommunikation, die in erster Linie der Aushandlung und Pflege von Beziehungen dienen. Darüber hinaus weist die Kommunikationsform medial bedingt Besonderheiten auf, die gleichfalls einen nicht unwesentlichen Einfluss auf das Interaktionsverhalten der Beteiligten haben (vgl. Imo 2015, 2019; Dürscheid/Frick 2014; mit sprachdidaktischem Fokus: Beißwenger 2016c, 2018). WhatsApp bringt die Struktur »klassischer « Chat-Anwendungen (Beißwenger 2007) mit der Mobilität von SMS-Dialogen und der Nutzung des Smartphones als Endgerät zusammen. ${ }^{2}$ Vom klassischen Chat unterscheidet sich die WhatsApp-Kommunikation weiterhin durch die Möglichkeit zum graduellen Wechsel zwischen einer asynchronen und synchronen Orientiertheit der Beteiligten auf das Kommunikationsgeschehen. Die persistente Verfügbarkeit des schriftlichen Verlaufsprotokolls entbindet die Nutzerinnen und Nutzer, sich für ihren Austausch $\mathrm{zu} »$ Chat-Sitzungen « zu verabreden; entsprechend kann jede/r Beteiligte Beiträge lesen und eigene neue Beiträge zur Kommunikation versenden, wann ihr oder ihm dies passend erscheint und genehm ist. Zusammenfassen lassen sich die Merkmale der durch WhatsApp konstituierten Kommunikationsform wie in Tab. 1 abgebildet (angelehnt an Imo 2015, S. 7; mit verschiedenen Erweiterungen und Präzisierungen).

Drei Merkmale der Kommunikationsform möchten wir ausführlicher kommentieren, da ihnen für die linguistische Analyse von WhatsApp-Kommunikation im Allgemeinen und von Emojis im Speziellen eine besondere Bedeutung zukommt:

\footnotetext{
1 https://de.statista.com/themen/1995/whatsapp/ [28.11.2019].

2 Die Möglichkeit, über die web.whatsapp.com die eigenen WhatsApp-Chats auch über Browsersoftware auf einem stationären PC zu bearbeiten, wollen wir hier nicht unerwähnt lassen, blenden wir als sekundäre Möglichkeit der Nutzung von WhatsApp aber in diesem Beitrag aus.
} 
Tab. 1 Merkmale der Kommunikationsform WhatsApp

\begin{tabular}{ll}
\hline Zeichentyp & Geschriebene Sprache; gesprochene Sprache; Bild; Video \\
Kommunikationsrichtung & Dialogisch \\
Kommunikationsstruktur & Sequenziell \\
$\begin{array}{l}\text { Anzahl der Kommunika- } \\
\text { tionspartner }\end{array}$ & Frei wählbar \\
Räumliche Dimension & Distanz \\
Zeitliche Dimension & $\begin{array}{l}\text { Kommunikationsbeiträge werden persistent protokolliert; das Kommuni- } \\
\text { kationsprotokoll ist allen Beteiligten jederzeit zugänglich, wodurch die Be- } \\
\text { teiligung an der und der Zugriff auf die Kommunikation für die einzelnen }\end{array}$ \\
& $\begin{array}{l}\text { Nutzer*innen individuell wählbar ist; entsprechend kann die Orientiertheit } \\
\text { der Beteiligten auf die Teilhabe am Kommunikationsgeschehen graduell }\end{array}$ \\
& $\begin{array}{l}\text { zwischen ,asynchron` und ,synchron` variieren. } \\
\text { Möglichkeit zum individuellen Wechsel der Beteiligungsaktivitäten (Produ- } \\
\text { zieren-Rezipieren) } \\
\text { Beteiligungsrollen }\end{array}$ \\
Kommunikationsmedium & $\begin{array}{l}\text { (Software:) App »WhatsApp« } \\
\text { (Realisierungsformat für Beiträge:) Textformen }\end{array}$ \\
\hline
\end{tabular}

\section{Dialogizität und Sequenzialität:}

Dialogizität und Sequenzialität sowie die Möglichkeit zur raschen Reaktion auf neue Beiträge der Partnerinnen und Partner rücken die WhatsApp-Kommunikation in strukturelle Nähe zur Interaktion. Zugrunde gelegt wird hier der von Imo (2013, S. 51-83) konzipierte Interaktionsbegriff, nach welchem Interaktion vorliegt, wenn zwei oder mehr Personen über Sprache eine Situation gemeinsam konstituieren, kontinuieren und modifizieren und die jeweiligen Äußerungen sequenziell aufeinander bezogen sind, so dass »schrittweise geteilter Sinn entsteht « (Imo 2013, S. 57). Die Beiträge der einzelnen Beteiligten sind dafür konzipiert, Relevanzverpflichtungen aus vorangegangenen Beiträgen anderer Beteiligter zu bedienen und zugleich den Kontext für Anschlussäußerungen zu bilden, die erneut von anderen realisiert werden.

\section{Bezug auf Textformen:}

Trotz ihrer strukturellen Nähe zur Interaktion ist für die WhatsApp-Kommunikation - wie auch für andere Formen internetbasierter Kommunikation - eine charakteristische Differenz zu mündlichen Gesprächen feststellbar, die sich auf allen Ebenen des Kommunikationsprozesses (Produktion, Organisation, Verarbeitung) auswirkt (Beißwenger 2007, 2016a) und die darauf zurückzuführen ist, dass in ihr Textformen als Mittel für die Produktion, die Fixierung und die Übermittlung von Beteiligtenäußerungen verwendet werden und die Verarbeitungsgrundlage für die Rezeption bereitstellen (Beißwenger 2020). Der Rückgriff auf Textformen als Format für die Realisierung von Kommunikationsbeiträgen hat technologiegeschichtliche Gründe (ebd.). Die gesellschaftliche Aneignung der dadurch konstituierten, neuen Form der Ermöglichung und Realisierung von dialogisch-sequenziell konzipierter, vermittelter zwischenmenschlicher Kommunikation hat eine Kommunikationspraxis hervorgebracht, in der die Begrenztheit der elementaren Interak- 
tionsform (= des mündlichen Gesprächs von Angesicht zu Angesicht) dadurch überwunden wird, dass (wie schon bei der Kommunikation mit Texten i.S. v. Ehlich 1983 et passim) Kommunikationsbeiträge persistent und von der Körperlichkeit der Beteiligten abgelöst werden. In WhatsApp und vergleichbaren Anwendungen wird Interaktion somit zeitflexibel und - aufgrund des Zugriffs über das Smartphone - darüber hinaus sogar mobil nutzbar und ubiquitär verfügbar. Die Möglichkeiten und die (zeitliche wie räumliche) Reichweite von Interaktion wird dadurch technologiespezifisch erweitert. Als Resultat ergibt sich eine neue Form der Organisation von Kommunikation, die wir mit Beißwenger (2020) als Textformen-basierte Interaktion bezeichnen.

Der Bezug auf Textformen als Format für die Produktion, Konstitution, Übermittlung und Bereitstellung von Beiträgen zu dialogisch-sequenziell intendierter Interaktion bildet die wesentliche Ressource, auf deren Hintergrund eine Indienstnahme von Bildzeichen als Mittel der Interaktionsgestaltung möglich wird. Charakteristisch für Textformen ist ihre Visualität und ihre Flächigkeit. Sie sind prototypischerweise (und auch historisch betrachtet) konzipiert als Träger geschriebener Sprache, die aber um weitere visuelle Zeichentypen (Schriftdesign, Bilder) angereichert sein kann und im Einzelfall auch aus ausschließlich nichtsprachlichen Einheiten (z. B. nur Bildern) bestehen können.

\section{Mobilität und Kontextbezug:}

Die Mobilität der Kommunikation führt darüber hinaus dazu, dass in jedweden Lebenslagen und an allen denkbaren Orten kommuniziert werden kann, »was zu neuen Herausforderungen des Kontextbezugs und der Indexikalisierung von Äußerungen geführt hat « (Imo 2019, S. 41-42). Durch die diatopische Struktur der Kommunikation bei der gleichzeitig gegebenen Möglichkeit, sich zeitnah dialogisch-sequenziell aufeinander zu beziehen, wird somit genau genommen nicht die Kommunikation als solche, sondern werden die einzelnen Beteiligten an der Interaktion individuell mobil: Sie wechseln ständig den Ort und den situativen Kontext, in dem sie sich individuell befinden, während sie mit ihren Interaktionspartnerinnen und -partnern über einen thematischen und sequenziellen Kontext verbunden sind und diesen dialogisch weiterspinnen. Das unterscheidet die Kommunikation in WhatsApp und vergleichbaren Anwendungen sowohl von mündlichen Interaktionen als auch vom sprachlichen Handeln mit Texten und wird nur möglich durch die Vermittlung über Textformen im oben beschriebenen Sinne.

Im Folgenden führen wir zunächst unseren Beschreibungsrahmen ein, der die Funktionen von Emojis auf dem Hintergrund der medialen, materialen und semiotischen Rahmenbedingungen beschreibt, die für die Kommunikation in WhatsApp charakteristisch und prägend sind (Abschnitt 2). Anschließend führen wir an einem Datenbeispiel aus dem MoCoDa2-Korpus vor, wie dieser Beschreibungsrahmen als Grundlage für die Formulierung von Funktionsbeschreibungen zu Emoji-Verwendungen im Gebrauch, also auf dem Hintergrund der dokumentierten oder rekonstruierten pragmatischen Bedingungen ihrer Verwendung, eingesetzt werden kann (Abschnitt 3). Der Beitrag schließt mit einem Ausblick dazu, weshalb Emojis als 
neue Mittel des sprachlichen Handelns gerade in privater Alltagskommunikation so erfolgreich sind (Abschnitt 4).

\section{Wie wir mit Emojis handeln: ein pragmatischer Beschreibungsrahmen}

In diesem Abschnitt stellen wir unseren Beschreibungsrahmen für die linguistische Analyse der Spezifik von Emojis in internetbasierter Kommunikation vor (vgl. ausführlich: Beißwenger/Pappert 2019a, S. 33-90). Der Ansatz ist pragmatisch fundiert. Die grundlegende Frage, auf die der Beschreibungsrahmen eine Antwort gibt und zu der er eine Reihe von Differenzierungen entwickelt, lautet »Welchen Beitrag leisten Emojis zur Organisation und Gestaltung von kommunikativem Handeln in Interaktion? « Die Spezifik des Handelns mit Emojis betrachten wir dabei als eine Disposition von Faktoren auf fünf Beschreibungsebenen:

(i) den medialen und materialen Bedingungen der Kommunikation im Internet, die für die Kommunikationsbeteiligten nicht änderbar sind und auf deren Hintergrund Emojis ihr spezifisches Handlungspotenzial entfalten können. Für die WhatsApp-Kommunikation zentral ist hier der schon in Abschnitt 1 beschriebene Bezug auf Textformen als Mittel der Konstitution, Vermittlung und Gestaltung von Verständigungshandeln.

(ii) den semiotischen Qualitäten, die sich aus der charakteristischen Zeichenhaftigkeit von Emojis ergeben und die in jeder Verwendung in spezifischer (und unterschiedlicher) Weise aktiviert werden können.

(iii) den pragmatischen Potenzialen, die sich unter den gegebenen Rahmenbedingungen (i) durch die semiotischen Qualitäten (ii) ergeben und die das Fundament dafür bilden, dass Emojis beim kommunikativen Handeln in der internetbasierten Kommunikation bestimmte Funktionen übernehmen können. Diese Potenziale liegen jeder Verwendung zugrunde und werden in jeder Verwendung eines Emojis ausgespielt.

(iv) den pragmatischen Funktionen, die Emojis in konkreten Verwendungen übernehmen und die sich in Relation zum sprachlichen und sequenziellen Kontext als Leistungen in Bezug auf die Handlungskonstitution und die Interaktionsgestaltung beschreiben lassen.

(v) den Praktiken, in die sich die pragmatischen Funktionen kontextabhängig ausdifferenzieren lassen und die als charakteristische Konstellationen aus medialen Ressourcen (= semiotischen Qualitäten und dadurch eröffneten Potenzialen) und den konkreten Anforderungen einer Interaktionssituation aufgefasst werden.

Abb. 2 veranschaulicht das Zusammenspiel der verschiedenen Faktoren. Für die funktionale Analyse von Emojis ist die oberste Ebene der Darstellung - die Ebene der konkreten Funktionen - von Belang. Hier werden konkrete Verwendungen von Emojis betrachtet und auf dem Hintergrund des situativen und sequenziellen Kontexts, in den sie eingebettet sind und der entweder dokumentiert ist (= Chatverlauf) oder anhand von Metadaten bzw. interpretativ erschlossen wird (= situative 


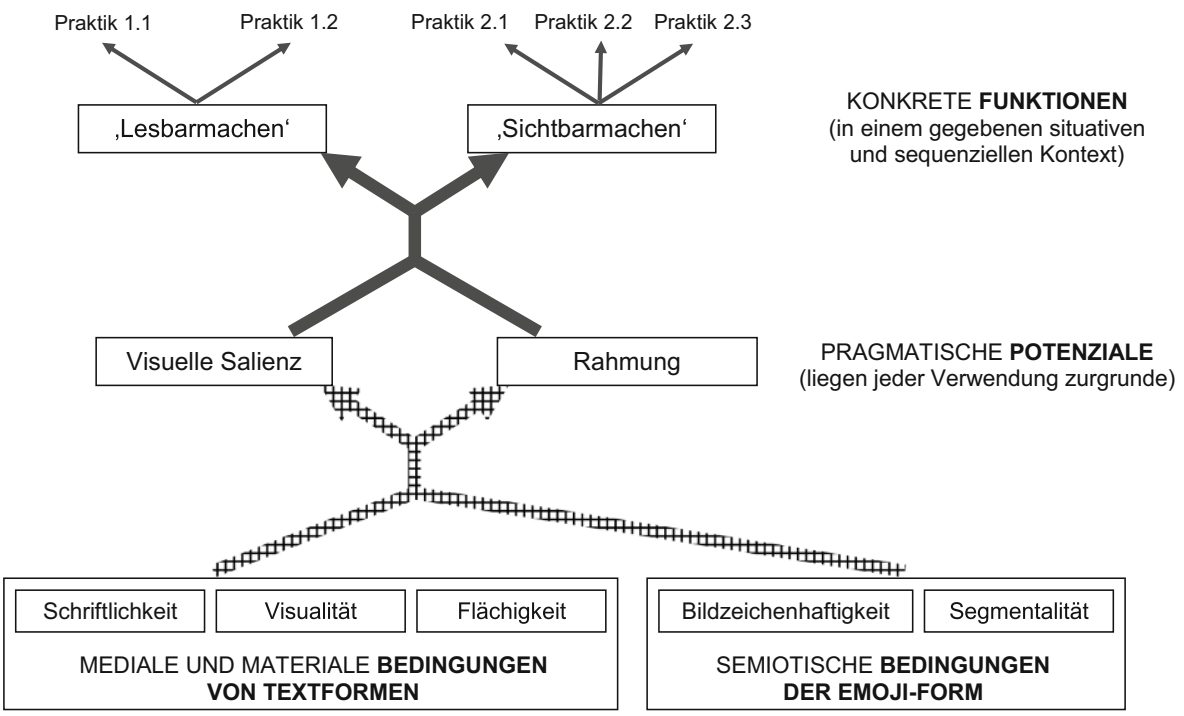

Abb. 2 Pragmatische Potenziale und Funktionsbereiche von Emojis (bezogen auf die WhatsApp-Kommunikation)

Bedingungen), mit einer Funktionsbeschreibung versehen. Um zu verstehen, weshalb Emojis in konkreten Kontexten bestimmte Funktionen übernehmen können und welche Möglichkeiten der Indienstnahme von Emojis für die Interaktionsgestaltung insgesamt existieren, sind die in Abb. 2 unterhalb der Ebene der Funktionen liegenden Beschreibungsebenen von Interesse. Die verschiedenen Ebenen stellen wir im Folgenden noch etwas ausführlicher vor.

\subsection{Mediale und materiale Bedingungen von Textformen}

Die Kommunikation per WhatsApp ist Textformen-basierte Kommunikation (Beißwenger 2020). Die Grundlage der Produktion, Übermittlung und Rezeption von Äußerungen bildet dasselbe Format, das auch dem sprachlichen Handeln mit monologischen Texten zugrunde liegt, das hier aber für die Organisation eines dialogischsequenziellen und somit interaktional intendierten Austauschs adaptiert wird. ${ }^{3}$ Das Format ist der Technologie genetisch (d.h. entwicklerseitig) eingeschrieben und für die Nutzerinnen und Nutzer im konkreten kommunikativen Handeln nicht änderoder variierbar. Das Format setzt u. a. die folgenden äußeren Bedingungen für die Beitragsgestaltung und für die Organisation von Kommunikation:

\footnotetext{
3 Mit Storrer $(2013,2018)$ kann die schriftliche, interaktional intendierte Kommunikation per Internet mit Blick auf die in ihr vorfindlichen sprachlichen Auffälligkeiten daher als Erweiterung der kommunikativen Möglichkeiten im Bereich der Nähekommunikation angesehen werden, die eine Schreibhaltung prominent setzt, die vor der internetbasierten Kommunikation bestenfalls randständig war und die von der Anpassung schriftsprachlicher Mittel an die Anforderungen und Bedingungen der Nähekommunikation geprägt ist (>interaktionsorientiertes Schreiben<, vgl. auch Beißwenger/Storrer 2012).
} 
- Es konzipiert kommunikative Äußerungen als Äußerungen, die den Adressaten als Produkte gegenübertreten und deren Produktion in ihrer Prozesshaftigkeit für die anderen Beteiligten zwar indiziert wird (»XY schreibt gerade...«), der Rezeption aber nicht zugänglich ist.

- Es konzipiert kommunikative Äußerungen als visuelle Einheiten, die prototypischerweise über geschriebene Sprache realisiert werden. Entsprechend ist die Verwaltung des user generated content, der von den Produzierenden zum Zwecke der Realisierung einer kommunikativen Äußerung über das User Interface eingegeben wird, zweidimensional und segmental vorstrukturiert: Inhalte werden über die Kombination diskreter visueller Segmente (Schriftzeichen und Bildzeichen) von links nach rechts konstruiert. An den Begrenzungen des Eingabefelds und der anhand der Eingaben vom System erzeugten Posting-Darstellung erfolgt ein automatischer Zeilenwechsel; die Produzierenden können auch selbst erzwungene Zeilenwechsel setzen. Die resultierenden Äußerungen sind als Ganze entlang der topologischen Dimensionen links-rechts und oben-unten gegliedert. ${ }^{4}$

- Die Nutzerinnen und Nutzer haben die Möglichkeit, in die Gestaltung ihrer Postings nicht nur per Tastatur erzeugte, geschriebene Sprache, sondern auch Medienobjekte einzubeziehen. Möglich ist die Einbindung von Bild- und Videodateien und von Emojis. ${ }^{5}$ Bild- und Videodateien können von den Produzierenden nicht frei platziert werden. Werden sie mit geschriebener Sprache kombiniert, stehen die sprachlichen Anteile des Postings grundsätzlich unterhalb des Medienobjekts. Emojis hingegen werden von den Produzierenden wie Schriftzeichen über die Tastatur erzeugt, die dafür vom Modus »Auswahl von Schriftzeichen« in einen Modus »Auswahl von Emojis« umgeschaltet werden muss. Eingegebene Emojis werden vom System strukturell analog wie Schriftzeichen behandelt, wodurch Emojis und Schriftzeichen bei der Eingabe frei kombiniert werden können (siehe Abb. 3).

\subsection{Semiotische Bedingungen der Emoji-Form}

Außerhalb konkreter Verwendungskontexte sind Emojis Bild-Zeichen, die Gesichtsausdrücke, Körpergesten, Tiere, Objekte, Symbole, Landschaften u. a. abbilden (s. die Beispiele auf der Emoji-Tastatur in Abb. 3). Im konkreten Verwendungskontext können diese Bilder auf ganz unterschiedliche Weise pragmatisiert werden. Dabei steht nicht das Emoji als Bild-Zeichen im Vordergrund, sondern der damit abgebilde-

\footnotetext{
4 Einen eigenen Typ von Posting stellen die sog. »Sprachnachrichten« dar, die in Form einer Audioaufnahme eingegeben und nach Beendigung der Aufnahme als Audiodatei übermittelt und über eine automatisch erzeugte, visuelle Schaltfläche am Bildschirm zum Abruf bereitgestellt werden. Auch in diesem Fall vermittelt eine flächige, visuelle Textform (die allerdings gegenüber dem Standard-Posting-Typ nicht vom Nutzer, sondern anhand eines Templates vom System erzeugt wurde) die vom Nutzer generierten Inhalte (= in diesem Fall eine Audioaufnahme, die typischerweise eine aufgesprochene Äußerung enthält) an die Adressatinnen und Adressaten.

5 Einen Sonderfall stellen die sog. »Sticker« und GIFs dar, die über eine entsprechende Tastatur eingefügt werden können, die sich aber im Unterschied zu Bild-, Audio-, Videodateien und Emojis nicht mit geschriebener Sprache in einem Posting kombinieren lassen. Äußerungen, die als Sticker oder als GIFs realisiert werden, stellen somit eigenständige Typen von Postings dar, die in diesem Beitrag nicht weiter berücksichtigt werden.
} 

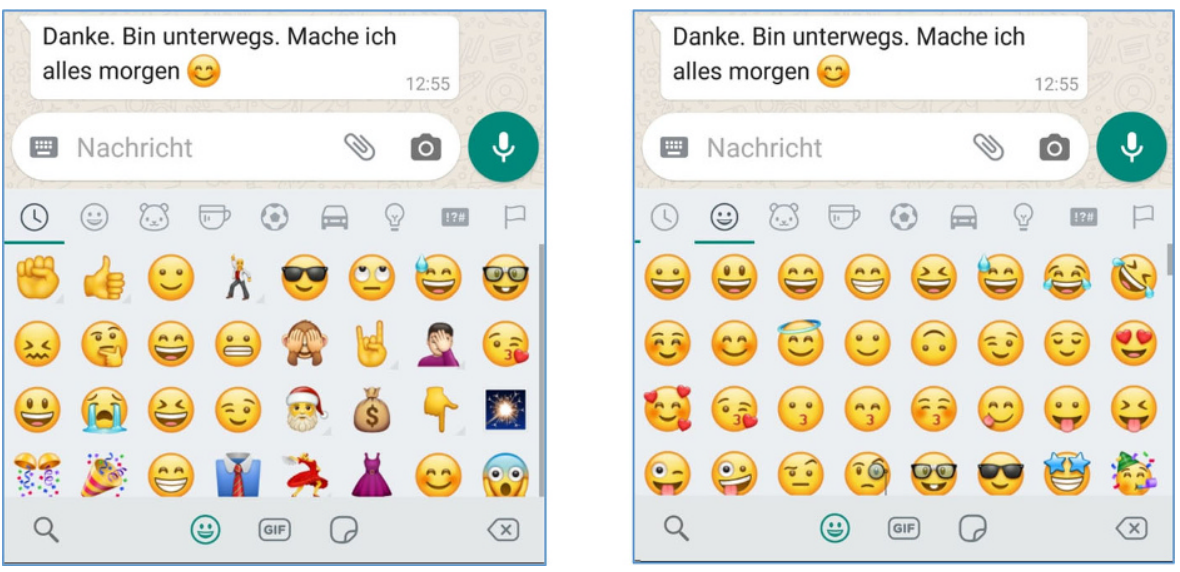

Abb. 3 Feld für die Eingabe einer neuen WhatsApp-»Nachricht« mit darunter aufgerufener Emoji-Tastatur. Der linke Screenshot zeigt die Tastatur mit den zuletzt vom Nutzenden verwendeten Emojis, der rechte Screenshot einen Ausschnitt aus der nach Emoji-Typen sortierten Standardansicht der Emoji-Tastatur. Durch Aktivierung des Tastatur-Icons, das im linken Bereich des Eingabefelds angezeigt ist, kann von der Emoji-Tastatur auf die Schrift-Tastatur umgeschaltet werden

te Gegenstand, der dann entweder als Ikon, als Symbol oder als Index genutzt wird. Diese Grundannahme illustrieren wir an zwei kurzen Beispielen, in denen EmojiFormen, die Gesichter, Gesichtsausdrücke bzw. gesichtsbezogene Gesten abbilden, einmal ikonisch, einmal indexikalisch und einmal symbolisch verwendet werden (siehe Abb. 4).

Bei den beiden Chatbeteiligten in Abb. 4 handelt es sich um Studierende eines Seminars zum Thema »Internetbasierte Kommunikation«, die sich gemeinsam auf eine mündliche Prüfung zu den Seminarinhalten vorbereiten. In Posting \#37 verwendet Lena das Äffchen mit den vor die Augen geschlagenen Händen indexikalisch, um auszudrücken, dass ihr der Sonnenbrand unangenehm ist (»au weia!«). In Posting \#40 verwendet Tobias ein weibliches Gesichts-Emoji mit dunkler Hautfarbe, um damit den von Lena berichteten Sonnenbrand wiederaufzugreifen. Das Emoji ist ikonisch verwendet und sichert zugleich die Kontinuität des Themas >Sonnenbrand <. In Posting \#41 verwendet Lena ein Emoji, das, als Form, ein nachdenkliches Gesicht nachbildet. Das deiktische »das « im sprachlichen Anteil des Postings kann auf zweierlei Weise interpretiert werden; je nach Lesart ergibt sich für das dreifach iterierte nachdenkliche Gesicht eine andere semiotische Qualität:

1. Wertet man »das« als anadeiktischen Rückverweis auf das Gesichts-Emoji mit dunkler Hautfarbe im Posting von Tobias, so lassen sich die nachdenklichen Gesichter von Lena als indexikalisch intendiert deuten: Lena möchte damit zum Ausdruck bringen, dass sie sich hinsichtlich der Validität ihrer Einschätzung, dass es sich bei dem gebräunten Emoji um ein indexikalisches Emoji handeln könne, unsicher ist (tatsächlich läge sie bei dieser Lesart mit dieser Einschätzung falsch). 
Abb. 4 https://db.mocoda2. de/\#/view/WzmhV

\title{
Lena \\ Und hab voll den Sonnenbrand \\ \#37 15:45
}

Tobias

Hast du noch draußen gesessen?

\#38 15:45

\begin{abstract}
Lena nichtmal 2 Stunden in der Sonne sitzen

\#39 15:46
\end{abstract}

$\mathrm{Ne}$, aber ich bin der weißeste Mensch der Welt, kann noch

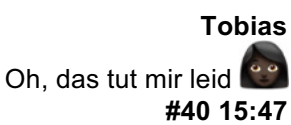

Lena

Das könnte ein indexikalischer Emoji sein \#41 15:48

2. Wertet man »das« hingegen als katadeiktischen Vorverweis auf die nachdenklichen Gesichter, so wäre $\because$ als eine metakommunikative Verwendung zu deuten, mit der das Emoji - und zwar in dreifacher Ausfertigung - als Form zitiert wird, die in diesem Fall ikonisch für sich selbst steht. Lesart 1 ist unter sequenzieller Perspektive wahrscheinlicher als Lesart 2; wir führen Lesart 2 dennoch auf, da sich an diesem Beispiel sehr schön zeigen lässt, dass je nach Interpretation des sprachlichen Kontexts für ein und dieselbe Emoji-Verwendung u. U. unterschiedliche zugrundeliegende Semiotisierungen angenommen werden können.

Als semiotische Bedingung der Emoji-Form werten wir, bezogen auf WhatsApp, auch die Tatsache, dass Emojis bei der Produktion von Kommunikationsbeiträgen frei miteinander und mit Schriftzeichen kombiniert werden können (Segmentalität). Sie sind segmentale Einheiten der Äußerungsgestaltung, die mit Einheiten, für die dieselben Kombinationsbedingungen gelten, zu größeren Einheiten kombiniert werden können.

\subsection{Pragmatische Potenziale: Visuelle Salienz und Rahmung}

Textformen-basierte Interaktion ist Interaktion unter den Bedingungen von Schriftlichkeit, Visualität und Flächigkeit. Persistenz, Visualität und Flächigkeit konstituieren ein Äußerungsformat, das sämtliche Äußerungsbestandteile in der Rezeption (grundsätzlich auch schon auf der Ebene der Produktion) simultan kopräsent präsentiert. Zeitlichkeit wird unter den Bedingungen von Textformen als »Sichtbarkeit in der Fläche« greifbar (Schmitz 2011, S. 30). Flächigkeit ermöglicht es, schriftliche Elemente räumlich zu komponieren, mit visuellen Mitteln der Graphostilistik und des Layouts zu arbeiten, wobei die Schrift gemeinsam mit anderen visuell basierten Zeichentypen erscheinen kann oder auch andere Zeichentypen gänzlich ohne Beteiligung von Schrift ein Kommunikat bilden können. Dennoch sind in den allermeisten 
Chatverläufen in WhatsApp die überwiegenden Äußerungsanteile schriftlicher Art. Geschriebene Sprache bildet somit die Default-Modalität, in der uns Beiträge zur Interaktion gegenübertreten. Vor diesem Hintergrund springen Bildzeichen unmittelbar ins Auge und binden - intendiert oder nichtintendiert - die Aufmerksamkeit von Rezipierenden.

Unter den Bedingungen (i) des Bezugs auf Textformen und (ii) der grundsätzlichen Bildzeichenhaftigkeit von Emojis (als Formen) ist ein fundamentales Charakteristikum von Emoji-Verwendungen in WhatsApp-Postings bzw. -Verläufen ihre visuelle Salienz: Sie sind Oberflächenerscheinungen, die sich vor dem Hintergrund des Geschriebenen für das Auge als Figur abheben. Sie werden unmittelbar wahrgenommen und holistisch verarbeitet, ohne dass - wie bei den schriftlichen ÄuBerungsanteilen - die Bedeutung und der Sinn erst durch Erlesen für die mentale Verarbeitung zugänglich gemacht werden kann. Bilder sind »wahrnehmungsnahe Zeichen « (Sachs-Hombach 2003, S. 74), die nicht nur auf den ersten Blick auffallen, sondern als »schnelle Schüsse ins Gehirn« (Kroeber-Riel 1996, S. IX) auch unmittelbar und mühelos interpretiert werden können.

In konkreten Interaktionszusammenhängen erweist sich die visuelle Salienz als pragmatisches Potenzial, das sich in jeder Verwendung eines Emojis aktualisiert eben weil deren Hervorstechen die Aufmerksamkeit der Rezipierenden, noch vor der Verarbeitung der sprachlichen Äußerungsanteile, auf bestimmte Stellen in den flächig ausgebreiteten Äußerungen und im ebenfalls flächig repräsentierten Interaktionsverlauf lenkt.

Emojis stechen allerdings nicht nur im wahrnehmungsphysiologischen Sinn heraus. Sie sind darüber hinaus in der Lage, Bewertungen über den sozialen Kontext von Interaktion zu indizieren. Sie zeigen an, dass ihre Verwenderin oder ihr Verwender die sozial-situativen Bedingungen der aktuellen Kommunikation als Nähedingungen konzipiert. Ihr Effekt ist der einer kontextschaffenden Rahmung (i. S. v. Auer 1999, S. 171, zur Rahmung vgl. auch Goffman 1977), bei der es darum geht, »eine informelle Atmosphäre unter Gleichen herzustellen« (Auer 1999, S. 173). Emojis markieren die Interaktion als (eher) informell und stellen den Adressat*innen den Schlüssel bereit, dessen sie bedürfen, um die Goffman'sche Frage »Was geht hier eigentlich vor? «(Goffman 1977, S. 16) für ihr Gegenüber, das in seinen Äußerungen Emojis verwendet, wie folgt zu beantworten: »eine Kommunikation, die - aus meiner Sicht - unter Personen geführt wird, die sich mindestens in dieser Situation als ungefähr Gleiche wahrnehmen, die sich wechselseitig wohlgesinnt sind, sich mit ihren Kompetenzen, Bedürfnissen und Wünschen wechselseitig respektieren und wertschätzen, und in deren Rahmen ggf. auch Privates zur Sprache kommen kann«.

\subsection{Funktionen und Praktiken}

Visuelle Salienz und Rahmung sind Effekte, die der Verwendung eines Emojis unter den medialen und materialen Bedingungen der WhatsApp-Kommunikation aufgrund ihrer Zeichenhaftigkeit und ihrer Zuordnung zur Sphäre des Informellen und Privaten unmittelbar anhaften und die sich nicht unterdrücken lassen. Aus diesem Grund betrachten wir sie nicht als Funktionen, sondern als Potenziale, die immer aktiv sind, sobald Emojis nicht die Default-Modalität der Äußerungsgestaltung bilden und 
sich somit figürlich vom Hintergrund abheben. ${ }^{6}$ Von einer Funktion sprechen wir dann, wenn der Verwendung eine Zweckmäßigkeit unterstellt werden kann, die sich als Alternative aus mehreren wählbaren Optionen beschreiben lässt. Trotzdem sind Funktionen nicht unabhängig von den beschriebenen pragmatischen Potenzialen: Salienz und Rahmung stellen die Ressourcen bereit, die für verschiedene und je spezifische Funktionen in Dienst genommen werden können.

Unter einer Funktion verstehen wir eine Disposition aus (i) Anforderungen, die sich übergreifend zu konkreten Kommunikationssituationen als Anforderungen der Interaktionsgestaltung ergeben, und (ii) dem wiederholten, in zahlreicher und kontextübergreifender Verwendung bezeugten Einsatz verfügbarer semiotischer und pragmatischer Ressourcen zu deren Bewältigung. Eine Funktion ist auf Ressourcen bestimmten Typs bezogen. Sie beschreibt deren empirisch nachvollziehbare Leistung bei der Bewältigung von Anforderungen eines bestimmten Typs.

Unter den Bedingungen konkreter Kontexte können Funktionen unterschiedliche Ausprägungen annehmen. Diese Ausprägungen beschreiben wir - in der Tradition von Schegloff (1997), Heritage (2010) und anderen - als Praktiken. Praktiken »zeichnen sich durch den kontextsensitiven Einsatz von bestimmten sprachlich-kommunikativen Formen als Ressourcen zur Lösung grundlegender Aufgaben der Interaktionskonstitution und zur Herstellung bestimmter Handlungen aus« (Deppermann et al. 2016, S. 1).

In Bezug auf Emojis unterscheiden wir zwei Funktionsbereiche, denen jeweils eine Reihe von Praktiken zugeordnet werden können. Der erste Funktionsbereich ist die Verwendung von Emojis als Lesbarmacher für sprachliche Äußerungen. Lesbarmachen ist Kontextualisierung unter den Bedingungen Textformen-basierter Interaktion. Mit der Verwendung von Emojis in dieser Funktion wird für die Adressatinnen und Adressaten der Kontext konstituiert, vor dessen Hintergrund und unter dessen Bedingungen der oder die Verwendende eine sprachliche Äußerung interpretiert wissen möchte oder anhand dessen die Einstellung der oder des Verwendenden zu einem geäußerten Sachverhalt rekonstruierbar gemacht werden soll, ohne diese explizit sprachlich zu formulieren. In dieser Verwendung fungieren Emojis als Kontextualisierungshinweise (i.S.v. Gumperz 1982) bzw. »Kontextualisierungsschlüssel« (Knoblauch 1991, S. 453), anhand derer der »Sinn der Mitteilung und die von Sprechern verfolgten Strategien und Intentionen« (ebd.) inferierbar werden sollen, ohne dass der Sinn, die Strategien und Intentionen von der oder dem Verwendenden explizit sprachlich präsentiert werden.

Eine sprachliche Äußerung wird um Lesbarkeitshinweise erweitert, wenn für den oder die Produzierende/n nicht gesichert ist, dass auf Grundlage der gewählten sprachlichen Form im gegebenen Kontext die Adressatinnen und Adressaten in der Lage sind, die Intentionen und Ziele ihrer bzw. seiner sprachlichen Handlung zu erkennen. Die Nichtexplizitheit der sprachlichen Äußerung kann pragmatisch be-

\footnotetext{
6 Die Default-Modalität kann in WhatsApp-Verläufen stellenweise von »Schrift« zu »Emoji« wechseln. Das ist dann der Fall, wenn über mehrere Postings ausschließlich oder überwiegend nur mit Emojis kommuniziert wird. Das einzelne Emoji hebt sich dann nicht mehr als saliente Gestalt vom Hintergrund ab; entsprechend kann das Potenzial der visuellen Salienz dann nicht in gleicher Weise ausgespielt werden wie im Fall von Verläufen, in denen das Geschriebene dominiert.
} 
gründet, d.h. bewusst gewählt sein. Lesbarkeitshinweise steuern die Interpretation. Sie erzeugen Inkonsistenzen, die signalisieren sollen, dass das sprachlich Geäußerte nicht das Gemeinte ist und dass nach einer Lesart der Äußerung zu suchen ist, die sich konsistent zum gegebenen Hinweis und zum sequenziellen Kontext verhält (Praktik 1.1: Kalkulierte Inkonsistenz als Anweisung zur Suche nach dem Gemeinten) oder sie legen die Einstellung des/der Produzierenden zum Geäußerten oder zu präferierten Folgehandlungen offen, die diese/r für die Interpretation des Geäußerten und des damit Gemeinten für wichtig hält (Praktik 1.2: Nichtredundante Markierung von Einstellungen als Präsentation von Innerlichkeit).

Den zweiten Funktionsbereich bildet die Verwendung von Emojis als Sichtbarmacher. Beim Sichtbarmachen geht es darum, die sprachliche Äußerung mit visuellen Mitteln anschaulich, gefällig und sozialverträglich zu gestalten. Praktiken des Sichtbarmachens haben gemeinsam, dass mit der Emoji-Verwendung zum Ausdruck gebracht wird, dass der oder die Verwendende die Beziehung zu den Adressatinnen und Adressaten als wertvoll, bewahrens- und schützenswert betrachtet. Im Gegensatz zu Praktiken des Lesbarmachens tragen Praktiken des Sichtbarmachens nichts zur Interpretation der Äußerung auf Ebene der Proposition oder Illokution bei. Praktiken des Sichtbarmachens markieren auch keine Einstellungen der Praktizierenden zu dem von ihnen Geäußerten. Stattdessen wird mit ihnen die Rahmungsleistung von Emojis unter Indienstnahme ihrer visuellen Salienz sowie der Formspezifik der konkret gewählten Emoji-Instanzen um weitere Aspekte der Beziehungsarbeit erweitert: Die Äußerung wird »ins Bild gesetzt«, d.h. Propositionen oder Teile davon werden - entweder zusätzlich zu ihrer sprachlichen Realisierung oder alternativ da$\mathrm{zu}$, in keinem Fall aber zwingend - in eine andere Zeichenmodalität transformiert, um anzuzeigen, dass man sich Mühe gemacht hat, die Äußerung für sie ansprechend zu gestalten und ihre Rezeption zu unterstützen. In diesen Verwendungen fungieren Emojis als Illustrationen (Praktik 2.1: Kalkulierte Redundanz als »Ins-Bild-Setzen« der sprachlichen Äußerung) oder als alternative (bildhafte) Realisierungsformen von Propositionen oder Teilpropositionen (Praktik 2.2: Handeln ohne Sprache).

Für die störungsfreie Gestaltung von Interaktion relevant werden Praktiken des Sichtbarmachens auch da, wo die Beziehung zu den Partnerinnen und Partnern durch potenzielle Effekte einzelner Handlungen in Gefahr gerät, gestört zu werden. In solchen Fällen fungieren Emojis als ins Bild gesetzter »sozialer Kitt«, der riskanten Äußerungen vorsorglich beigefügt wird, um anzuzeigen, dass trotz möglicherweise unerwünschter Implikationen der Äußerung die Verwendenden die Wünsche der Adressatinnen und Adressaten respektieren und auf eine gute und von wechselseitiger Wertschätzung geprägte Beziehung achten. In solchen Verwendungen sind Emojis Mittel des höflichen Handelns und dienen der Abschwächung potenzieller Gesichtsbedrohungen (Praktik 2.3: Abschwächen als Mittel zur sozialverträglichen Organisation des sprachlichen Handelns). Trotz Abschwächung bleibt das, was mit der Äußerung proponiert und bezweckt wird (z. B. jemanden zu etwas auffordern, jemanden kritisieren), unverändert. Das unterscheidet das Sichtbarmachen zum Zwecke der sozialverträglichen Gestaltung der eigenen Äußerung von Praktiken des Lesbarmachens, mit denen entweder eine andere Lesart nahegelegt wird oder mit denen Einstellungen zum Proponierten markiert werden, die an den sprachlichen Äußerungsanteilen selbst nicht abgelesen werden können. 


\section{Analyse}

In der folgenden Analyse werden wir den vorgestellten Beschreibungsrahmen auf die Analyse eines längeren Ausschnitts aus einem WhatsApp-Chat anwenden. Dabei werden wir zeigen, dass die herauszuarbeitenden Funktionen und Praktiken immer in Bezug auf den interaktiven Aushandlungsprozess zu bestimmen sind. Entscheidend für die Interpretation konkreter Emoji-Verwendungen ist die Platzierung des Postings, in das sie eingebettet sind, innerhalb des Verlaufsprotokolls, das - zusammen mit den sprachlichen Anteilen des einbettenden Postings - den sequenziellen Kontext und den Äußerungskontext bildet, vor dessen Hintergrund das Emoji als Ausdruck einer bestimmten Funktion (Lesbarmachen oder Sichtbarmachen) und einer spezifischen Praktik zu bestimmen ist.

Am Beispiel wollen wir zeigen, dass konkrete Verwendungen von Emojis mit dem in Abschnitt 2 skizzierten Beschreibungsrahmen funktional beschreibbar sind. Zugleich zeigen wir aber auch, dass die Frage, was ein Emoji im Einzelfall leistet, in hohem Grade davon abhängig ist, was wir als Analysierende über die Beteiligten, ihre Beziehung und ihre kommunikative und biographische Vorgeschichte wissen. Wir werden diese Unsicherheiten da, wo sie analyserelevant sind, explizit thematisieren, weil sich je nach Vorannahme unterschiedliche Lesarten - und damit unterschiedliche Funktionszuschreibungen - für einzelne Emoji-Verwendungen ergeben können. Das macht die Analyse nicht weniger informativ, aber weniger spekulativ. ${ }^{7}$

In dem in Beispiel 2 wiedergegebenen Datenbeispiel aus dem MoCoDa2-Korpus geht es um nachträgliche Geburtstagsgrüße und den Versuch einer Verabredung. Für die Interaktionsbeteiligten Karina und Justin ist in der MoCoDa2 dokumentiert, dass sie aus dem gleichen Heimatdorf stammen, mittlerweile aber beide in Münster leben und schon lange Zeit befreundet sind. Sie sehen sich allerdings sehr selten, was offenkundig in erster Linie an Justin zu liegen scheint, was durch den weiteren (hier nicht wiedergegebenen) Chatverlauf gestützt wird. Der hier analysierte Ausschnitt, dessen Beiträge alle am gleichen Tag verfasst wurden, umfasst zwölf Postings, die zwanzig Emoji-Verwendungen enthalten.

Wir präsentieren zunächst den Ausschnitt als Ganzen (Abb. 5) und analysieren anschließend sukzessive die Emoji-Verwendungen in den Postings. Um den Nachvollzug der Analyse zu erleichtern, stellen wir unseren Analysetexten die jeweils analysierten Postings noch einmal zusätzlich voran.

In Posting \#1 (Abb. 6), das um 1:34 Uhr versendet wurde, beglückwünscht Justin Karina leicht verspätet zum Geburtstag. Dabei weist er darauf hin, dass er versucht habe, - wohl pünktlich - anzurufen und zitiert seine für das Telefonat geplante Formulierung »Happy birthday du Nudel ey«, wobei er die im Gespräch erwartbare konzeptionelle Mündlichkeit reproduziert. Dem Glückwunsch beigefügt werden

\footnotetext{
7 Die Abhängigkeit linguistischer Aussagen zur Funktion sprachlicher Einheiten von der Verfügbarkeit von dokumentiertem Kontextwissen gilt selbstverständlich nicht nur für die Analyse digitaler, sondern von jedweder Art von natürlicher Kommunikation.
} 
Abb. 5 https://db.mocoda2. de/\#/view/53uBo
Mo. 23. Apr. 2018

\section{Justin}

Ich wollts eig am Telefon sagen „Happy birthday du Nudel ey“ (1) Hoffe du hattest einen schönen Tag.

Ich hab Hausarbeit gechrieben und bis grad gearbeitet und sitze grad wieder am Screibtisch! Deswegen melde ich mich erst jetzt

\#1 01:34

Karina

Dankiiiiiiiii Ich hatte kurz versucht zurück zu rufen aber da warst du sicher arbeiten

\#2 11:42

\section{Justin}

Ja misty ey. Wann sehen wir uns? Bin grad aufm weg zur Uni

um meine letzte richtige Hausarbeit abzugeben

\#3 13:46

Karina

Geilooooon

\#4 14:15

Karina

Mir egal, hab eigentlich viel Zeit diese Woche

\#5 14:15

\section{Justin}

Ok ich nicht

\#6 14:20

Justin

Ehm

\#7 14:21

Karina

Like always

\#8 14:21

\section{Justin}

Müsste sponti gucken. Hätte Mittwoch nach der uni ab $13 \mathrm{Uhr}$ Zeit bis 16 Uhr

\section{\#9 14:21}

Justin

Sorry

\#10 14:22

Justin

Ich bin sowas von Urlaubsreif

\#11 14:25 
ein freundlich lachendes Emoji $(\vartheta$ ) sowie eine dreifache Iteration eines anderen Emojis, das eine spanische (?) Tänzerin abbildet (

Für die Deutung des lachenden Emojis bieten sich drei verschiedene Lesarten an. Welche zutreffend ist, hängt davon ab, (a) auf welchen Teil der vorgängigen Äußerung sich das Emoji bezieht und (b) was wir als Ergebnis gemeinsamer Biographie und Kommunikationsgeschichte der beiden voraussetzen können oder annehmen wollen.

Die Lesarten 1 und 2 deuten das lachende Emoji als Ausdruck einer Praktik des Sichtbarmachens, und zwar im Dienste des face work (= Praktik 2.3). Bezieht sich das Emoji auf den erfolglosen Anruf, würde es der Gesichtswahrung beider Beteiligter dienen: zum einen der Wahrung von Justins Gesicht, weil er es nur einmal versucht hat, was angesichts des Geburtstages einer (engen?) Freundin durchaus als unangemessen aufgefasst werden kann, zum anderen der Wahrung von Karinas Gesicht, die ganz offensichtlich nicht erreichbar war (= Lesart 1). Bezieht sich das Emoji hingegen auf die Bezeichnung »Nudel« würde das positive Selbstbild Karinas bestätigt, das durch die unkommentierte Verwendung des Ausdrucks »Nudel« scheinbar angegriffen wird (= Lesart 2). ${ }^{8}$ Denkbar wäre aber auch, dass das freundlich lachende Emoji sich auf die recht ungewöhnliche Bezeichnung »Nudel« bezieht. Es macht diese insofern lesbar, als durch das Emoji darauf hingedeutet wird, dass »Nudel« hier scherzhaft gemeint ist (= Lesart 3 und Praktik 1.1). Alternativ wäre auch denkbar, dass »Nudel« eine über die Zeit in der Kommunikationsgeschichte der beiden geronnene zärtliche Bezeichnung für Karina darstellt. In diesem Fall läge erneut eine Praktik des Sichtbarmachens vor: Das Emoji bringt keine zusätzlich zum Sprachlichen relevanten Interpretationshinweise ins Spiel, da Karina davon ausgehen kann, dass Justin, wenn er sie »Nudel« nennt, dies zärtlich meint. Stattdessen wird über die Hinzufügung eines positiven Gesichtsausdrucks das positive Gefühl, das Justin Karina mit der Verwendung des Ausdrucks »Nudel« entgegenbringt, ins Bild gesetzt (= Praktik 2.1). Die Redundanz des Bildlichen zum sprachlich Geäußerten ist damit ein Ausdruck der Wertschätzung (= Lesart 4).

Die drei Tänzerinnen setzen den Geburtstag gleichsam als schwungvollen Tanz ins Bild und schmücken als Praktik des Sichtbarmachens den Glückwunsch zusätzlich aus (= Praktik 2.1). Die nachfolgende Hoffnungsbekundung (»Hoffe, du hattest einen schönen Tag.«) wird ergänzt um zwei abgebildete Herzen, die an dieser Stelle symbolisch - also unter Aktivierung von kulturellem Hintergrundwissen zur Konvention über Herz-Symbole - als Ausdruck und Bestätigung von Wertschätzung gegenüber Karina gedeutet werden können. Als Praktik machen sie die innige Beziehung sichtbar, wobei das »Doppelherz« diesen Effekt noch verstärkt (= Praktik 2.2); zugleich kann das Symbol, ebenfalls im Rahmen von Praktiken des Sichtbarmachens, auch als Ins-Bild-Setzung der Intensität verstanden werden, mit der Justin Karina sprachlich wünscht, einen schönen Tag gehabt zu haben (= Praktik 2.1).

Unabhängig von ihrer konkreten Funktion - und auch unabhängig davon, ob Justin das intendiert hat oder nicht - wirken die beiden Herzen zudem gliedernd, indem

8 Zum face work mit Emojis vgl. Beißwenger/Pappert (2019b, 2019c). 
Abb. 6 Posting \#1 aus Abb. 5

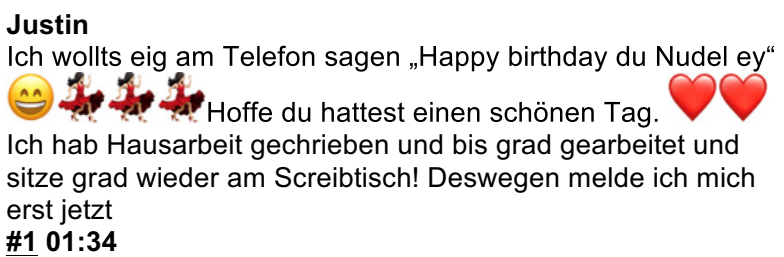

sie zwei aufeinanderfolgende sprachliche Handlungen voneinander abgrenzen, und zwar auf sehr markante Weise. Hier kommt das pragmatische Potenzial der visuellen Salienz von Emojis zum Tragen, das Emojis immer dann ausspielen können, wenn ihre visuelle Umgebung mehrheitlich nicht aus Grafiken (sondern wie hier aus Schriftzeichen) besteht.

Die Analyse der Emojis im Posting \#1 (Abb. 6) zeigt, dass dem Sichtbarmachen, also der Beziehungsarbeit, für Justin in seiner Kommunikation mit Karina eine wichtige Rolle zukommt und dass er die situativen Rahmenbedingungen der Kommunikation mit Karina als Nähebedingungen konzipiert; ansonsten würde er keine positiven Gefühle indizierende und stark emotional konnotierte Emoji-Formen verwenden. Dass er den Austausch als Nähekommunikation konzipiert, wird durch Indikatoren bei der sprachlichen Gestaltung gestützt: Das Posting weist eine Reihe von Merkmalen konzeptioneller Mündlichkeit auf, etwa die Interjektion »ey «, einen parataktischen Strukturaufbau sowie die für die gesprochene Umgangssprache typischen Elisionen unbetonter Vokale (»hab«, »grad «) und eine ebenfalls umgangssprachliche Verschmelzungsform (»wollts«) (vgl. Beißwenger 2016b, S. 22).

In Posting \#2 (Abb. 7) präsentiert Karina als erstes den sequenziell voraussehbaren Dank für die Glückwünsche - zwar in der Form eher ungewöhnlich aufgrund der Auswahl des Vokals [i], aber ebenso wie die Äußerungen Justins angelehnt an die gesprochene Umgangssprache, hier realisiert mittels einer über die Iteration des Vokalgraphems »emulierten Prosodie« (Haase et al. 1997, S. 68). Begleitet wird der Dank durch zwei Herz-Emojis

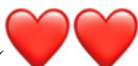

Herzen spiegeln die Visualisierung des Beziehungsstatus (bzw. die Ausschmückung des schönen-Tag-Wunsches) von Justin zurück (= Praktik 2.1 bzw. 2.2). Das Sonnen-Emoji lässt wiederum zwei verschiedene Deutungen zu, je nachdem, welches sprachliche oder nichtsprachliche Element des Kontextes man als Bezugseinheit annimmt: Geht man davon aus, dass die Sonne auf dem unmittelbar vorangegangenen »Doppelherz«-Symbol operiert, so kann es als symbolischer Verstärker für die mit den Herzen realisierte Form der Beziehungspflege aufgefasst werden. Die Sonne steht an der Stelle konventionell für (Lebens-)Freude und Wärme, und charakterisiert auf diese Weise die Art der Beziehung, zumindest soweit sie Karina betrifft (= Lesart 1). Es stellt eine Form des »Handelns ohne Sprache« dar, mit der die Beziehung selbst thematisiert wird (= Praktik 2.2). Alternativ lässt sich das SonnenEmoji aber auch auf den von Justin in Posting \#1 (Abb. 6) gewünschten »schönen Tag« beziehen. Zur stereotypen Vorstellung von einem schönen Tag gehört üblicherweise schönes Wetter, und schönes Wetter wird kulturell eingespielt (und z.B. auch auf Wetterkarten so reproduziert) mit dem Symbol einer scheinenden Sonne 
Abb. 7 Posting \#2 aus Abb. 5

Karina

Dankiiiiiiii

Ich hatte kurz versucht zurück zu rufen

aber da warst du sicher arbeiten

\#2 11:42

dargestellt. In dieser Lesart handelte es sich bei der Verwendung des Sonnen-Emojis um eine bildliche Referenz auf das Thema des von Justin geäußerten Wunsches; die Sonne sichert somit thematische Kontinuität und macht deutlich, dass sich Karinas »Dankiiiiiiiii« nicht darauf bezieht, dass sich Justin »erst jetzt wieder « meldet (= den letzten Teil seines Postings), sondern auf den Wunsch für einen schönen Tag (der im Vorgänger-Posting etwas weiter zurückliegt). Das Sonnen-Emoji sichert in diesem Fall den Bezug und ersetzt eine sprachliche Form der Bezugnahme (= Lesart 2); es kann in dieser Lesart ebenfalls der Praktik »Handeln ohne Sprache «(= Praktik 2.2) zugeordnet werden, hier allerdings realisiert in Form der Wahl einer bildlichen anstelle einer sprachlichen Referenz.

Die darauf folgende Äußerung im gleichen Posting (»Ich hatte kurz versucht zurück zu rufen aber da warst du sicher arbeiten«) wird durch Karina mit zwei gesichtsdarstellenden Emojis versehen: einem freundlich lachenden $(\hat{A})$ und einem »zähnelachenden« $(\because)$. Das erste der beiden Emojis kann als Abschwächer im Rahmen einer Face-work-Strategie gedeutet werden (= Praktik 2.3): Die Unterstellung, Justin sei »sicher wieder arbeiten « rekurriert auf gemeinsame Vorgeschichte; offenbar empfindet Karina es als generell schwer, Justin zu erreichen oder mit ihm in Kontakt zu treten, und offenbar hat dies immer - entweder faktisch oder nach dem, was er ihr als Gründe nennt - damit zu tun, dass er arbeiten muss. Mit dem zweiten Emoji bringt Karina ihre Betrübnis darüber zum Ausdruck, dass sie Justin nicht erreicht hat - oder sogar darüber, dass er generell schwer zu erreichen ist. Das erste Emoji dient somit dem Sichtbarmachen und der Beziehungspflege, das zweite Emoji liefert hingegen als Lesbarmacher eine Einstellungsbekundung zum sprachlich Proponierten und damit einen Interpretationshinweis für Justin, wie die Äußerung gemeint ist (= Praktik 1.2). Das lachende Emoji spiegelt darüber hinaus die erste Emoji-Verwendung von Justin in Posting \#1 (Abb. 6), die in einer der aufgezeigten Lesarten ebenfalls als Ausdruck von face work gedeutet werden kann; die Spiegelung könnte damit zugleich als Aufgreifen nicht nur derselben Form, sondern auch der damit realisierten Praktik (2.3) aufgefasst werden.

Alternativ könnten auch beide Emojis als Lesbarmacher (= Praktik 1.2) intendiert sein: Das erste bezöge sich dann auf die Proposition des ersten Teilsatzes (»Ich hatte kurz versucht zurück zu rufen «) und drückte Karinas Vorfreude darauf aus, Justin zu erreichen; das zweite Emoji bezöge sich, wie schon in der zuvor beschriebenen Interpretation, auf Karinas Betrübnis über Justins Nicht-Erreichbarkeit. Durch die Adjazenz der beiden Emojis würden die beiden Gefühlszustände von Karina zugleich unmittelbar miteinander kontrastiert; das Emoji-Paar illustriert Karinas »Wechselbad der Gefühle«. 
Abb. 8 Postings \#3 und \#4 aus Abb. 5

\section{Justin}

um meine letzte richtige Hausarbeit abzugeben

\#3 13:46

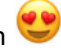

Karina Geilooooon

\#4 14:15

In Posting \#3 (Abb. 8) reagiert Justin auf die von Karina visuell nahegelegte innere Spannung, indem er diese sprachlich aufgreift (Responsiv »Ja ) und auch seinerseits Bedauern über seine (punktuelle oder generelle) Nichterreichbarkeit ausdrückt (»misty ey«). Die Form »misty« kann dabei ggf. als Spiegelung der von Karina einleitend zu ihrem Vorgänger-Posting gewählten Form »Dank $i \ll$ gedeutet werden. Um das Problem seiner Nichterreichbarkeit zu bearbeiten, setzt er ein mögliches künftiges Treffen relevant (»Wann sehen wir uns?«). Die Information, dass er gerade auf dem Weg zur Uni sei, um die letzte Hausarbeit abzugeben, könnte als Angebot für einen möglichen Termin aufgefasst werden oder als Hinweis darauf, dass er nun künftig wieder mehr Zeit haben werde. Das das Posting abschließende

Herzaugen-Emoji ( ) macht die überbordende Freude über die Abgabe lesbar, d.h. es signalisiert (nichtredundant) seine Einstellung zum sprachlich ausgedrückten Sachverhalt (= Praktik 1.2). Die damit ausgedrückte Freude wird von Karina in \#4 (Abb. 8) sowohl verbal als auch bildlich zurückgespiegelt. Abermals verwendet sie dazu eine über Graphemiteration konstituierte Form emulierter Prosodie und greift eine von Justin gewählte Emoji-Form spiegelnd auf. Während in Posting \#3 (Abb. 8) das Herzaugen-Emoji sich nichtredundant zur sprachlichen Äußerung verhält und dem Lesbarmachen dient, verwendet Karina in Posting \#4 (Abb. 8) dasselbe Emoji als Sichtbarmacher: Ihre positive Emotion zum von Justin geäußerten Sachverhalt wird im sprachlichen sowohl lexikalisch (»geil«) als auch durch die Emphasemarkierung (Iteration) ausgedrückt, ein zusätzlicher Interpretationshinweis ist eigentlich nicht erforderlich. Das Emoji dient hier der Ins-Bild-Setzung und damit dem Sichtbarmachen geteilter Freude (= Praktik 2.1).

Mit Posting \#5 (Abb. 9) erfüllt Karina die konditionelle Relevanz, die sich aus Justins Frage nach einem möglichen Treffen in Posting \#3 (Abb. 8) ergibt, und zeigt an, zeitlich sehr flexibel zu sein. Das damit unterbreitete Angebot, einen Termin wählen zu dürfen, wird von Justin in \#6 (Abb. 9) recht schroff zurückgewiesen. Er realisiert damit eine dispräferierte Folgehandlung, die sowohl sein eigenes als auch - und vor allem - das Gesicht Karinas bedroht. Diese Bedrohung wird abgefedert durch

das freundlich lachende Emoji ( $(A)$ ), das als Praktik des Sichtbarmachens zum Schutze beider Gesichter eingesetzt wird (= Praktik 2.3). Auch die in \#7 (Abb. 9) von Justin nachgeschobene Interjektion »Ehm« unterstützt das face work und soll vermutlich anzeigen, dass es Justin unangenehm ist, Karina (zum wiederholten Male?) zurückweisen zu müssen. Die Reaktion von Karina deutet darauf hin, dass die 
Abb. 9 Postings \#5 bis \#8 aus Abb. 5

\section{Justin \\ Ok ich nicht \\ \#6 14:20 \\ Justin \\ Ehm \\ \#7 14:21}

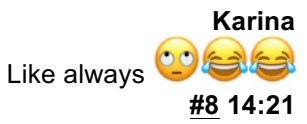

Nichtverfügbarkeit Justins eher die Regel als die Ausnahme darstellt, wovon die verbale Aussage »Like always « in \#8 (Abb. 9) zweifelsfrei Zeugnis ablegt.

Dass Karina über diese Zurückweisung nicht sonderlich erfreut ist, zeigt sie in Posting \#8 (Abb. 9). Zugleich signalisiert sie, dass Justins Reaktion für sie nicht unerwartet ist, indem sie auf Kontinuität zur Vorgeschichte verweist (»Like always«); der Wechsel ins Englische kann als Beitrag zum face work gedeutet werden (ei-

genes Gesicht schützen). Das augenrollende Emoji (-) illustriert ihre Frustration darüber, dass Justin sie - erwartungskonform - einmal wieder zurückgewiesen hat. Im sequenziellen Kontext ist klar, dass Karinas »Like always« Frustration ausdrückt; entsprechend wäre das Emoji als Sichtbarmacher (Ins-Bild-Setzen) zu werten (= Praktik 2.1). Die beiden unmittelbar angehängten tränenlachenden Emojis federn die Gefährdung beider Gesichter ab: des Gesichts Justins, dem unangenehm sein muss, dass er Karina erneut zurückgewiesen hat; des Gesichts Karinas, weil sie ein Angebot gemacht hat, das zurückgewiesen wurde (= Praktik 2.3). Das Beispiel illustriert erneut, dass die Abfolge von Emojis innerhalb einer Äußerung nicht notwendigerweise beliebig ist.

Justins Posting \#9 (Abb. 10) könnte laut Zeitstempel parallel zum davor angezeigten Posting \#8 (Abb. 9) von Karina verfasst worden sein. Darauf deutet das »inhaltliche Zurückrudern« Justins, der mittels angedeuteter Offerten verzweifelt versucht, den selbst erkannten Fauxpas aus \#6 (Abb. 9) auszubügeln. In \#10 (Abb. 10) folgt dann eine explizite Entschuldigung, die vermutlich als Replik auf Posting \#8 (Abb. 9) intendiert ist. Der über die gewählte Emoji-Form abgebildete weinerliche

Gesichtsausdruck ( $)$ setzt die die Entschuldigung erforderlich machende Empfindung ins Bild und verleiht der verbalen Äußerung (»Sorry«) Nachdruck bzw. emotionale Obertöne, derer man sich ob des visualisierten Gesichtsausdrucks wohl schwerer entziehen kann (= Praktik 2.1). Es folgt eine mehr oder weniger plausible Begründung des offenkundig überforderten Zustands Justins (\#11 (Abb. 10)). Karina ratifiziert Justins »Monolog « mit immerhin acht Minuten Differenz zu Justins erster Äußerung (\#9 (Abb. 10)) und dem darin offerierten Terminfenster (»Ok«), geht aber nicht auf Justins Terminvorschlag ein, sondern bietet an, die Verständigung über ein mögliches Treffen zu unbestimmter Zeit erneut aufzunehmen. Den beiden tränenla- 
Abb. 10 Postings \#9 bis \#12 aus Abb. 5

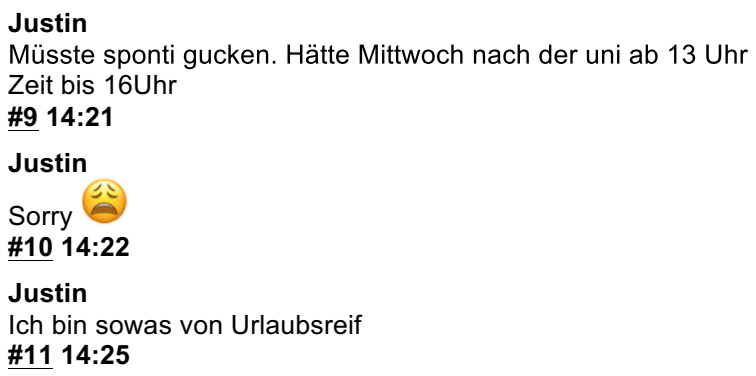

Karina können dann ja nochmal schreiben

chenden Emojis, die Karina ihrem »Ok« anfügt, können unterschiedliche Lesarten und Praktiken zugeordnet werden. Die sozialverträglichere der beiden Lesarten bestünde darin, die beiden Emojis als Ausdruck von Face-work-Praktiken zu werten, mit denen neben der verbalen Ratifizierung für Justins Überforderung Justin auf der Beziehungsebene zu verstehen gegeben werden soll »nicht schlimm, ich nehme dir das nicht übel« (= Praktik 2.3). Justin wird damit von Karina offeriert, trotz seines offensichtlichen Fauxpas' sein positives Gesicht zu wahren. In einer zweiten Lesart könnten die beiden Emojis allerdings auch als Einstellungsmarkierung - und damit als Lesbarmacher - gedeutet werden (Paraphrase: »Ich lache mich schlapp über deine ständigen Ausflüchte. Mehr als >Ok<fällt mir dazu nicht ein«) (= Praktik 1.2). In dieser Lesart könnte die Emoji-Wahl von Karina dadurch motiviert sein, nicht auf der sprachlichen Ebene unhöflich werden zu müssen und den Ball in Justins Feld zurückzuspielen, der nun selbst sehen soll, wie er ihre »schmallippige « Replik »Ok«, verbunden mit einem anschließenden Nicht-Eingehen auf sein in Posting \#9 (Abb. 10) vorgeschlagenes Terminangebot, interpretieren möchte. In dieser Lesart zeigt sich, dass Emojis mitunter auch deshalb als Ausdrucksmittel gewählt werden, um in Kommunikation zweckmäßig vage zu bleiben und gerade nicht sprachlich explizit zu werden; im vorliegenden Fall wäre das bedingt durch die Intention, das Näheverhältnis der beiden nicht ernsthaft gefährden zu wollen. Anstatt einen potenziell gesichtsbedrohenden Akt auszuführen entscheidet sich Karina dafür, das Thema zu vertagen und mit »guter Miene« (abgebildet durch die gewählten Emojis) die Kontinuität des Beziehungsstatus anzuzeigen (Rahmung).

\section{Fazit}

In diesem Beitrag haben wir einen Beschreibungsrahmen vorgestellt, der es ermöglicht, Emoji-Verwendungen in WhatsApp-Verläufen Funktionen in Bezug auf die zwei grundlegenden Aufgaben bei der Interaktionsgestaltung zuzuweisen: die Herstellung und Sicherung von Verstehen (Lesbarmachen) und die Beziehungspflege (Sichtbarmachen). Als Gestalten, die vor dem Hintergrund der medialen und materialen Bedingungen von Textformen Aufmerksamkeit binden, und als Bildzeichen,

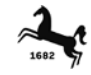


die bei der Wahrnehmung unmittelbar Assoziationen ermöglichen, können Emojis in beiden Bereichen wichtige Aufgaben übernehmen. Sie liefern nicht-sprachliche Interpretationshinweise zu sprachlichen Äußerungsanteilen, und sie können auf vielfältige Weise eingesetzt werden, um die Beziehung zum Gegenüber zu indizieren, zu thematisieren und gegen innere Gefährdungen abzusichern. Im vorliegenden Beitrag haben wir das exemplarisch an der Analyse eines längeren Ausschnitts aus einem WhatsApp-Chat illustriert (weitere Analysen finden sich in Beißwenger/Pappert 2019a).

Es greift daher zu kurz, Emojis lediglich als »Ersatz« für Gestik, Mimik oder Körpersprache zu betrachten. Stattdessen ermöglichen Emojis die Realisierung von Funktionen, die wir in Face-to-face-Begegnungen mit Mitteln der Körperlichkeit ausdrücken, die aber in digitaler Kommunikation nicht zur Verfügung stehen. Was in der digitalen Welt fehlt, ist somit nicht die Körperlichkeit als solche; stattdessen sind es Ressourcen, die unter den Bedingungen der digitalen Welt und insbesondere der Vermittlung durch Textformen Vergleichbares zu leisten imstande sind, und die sich als zweckmäßige Mittel für die Verstehenssicherung und Beziehungsarbeit erweisen. Dass Small Talk - in diesem Beitrag verstanden als Alltagskommunikation, die sich unter Bedingungen sozialer, in aller Regel auch zeitlicher, Nähe vollzieht und in welcher der Aushandlung und Pflege von Beziehung ein zentraler Stellenwert zukommt - auch über den Austausch von WhatsApp-Nachrichten effizient organisiert werden kann, ist nicht zuletzt (oder gerade) ein Verdienst von Praktiken, mit denen Emojis in den Dienst der Interaktionsgestaltung gestellt werden.

Eine spannende Aufgabe für künftige Forschungen zum Handeln mit Emojis wird es sein zu zeigen, inwieweit Konstellationen aus bestimmten Emoji-Formen und Funktionen bzw. Praktiken im wiederholten und massenhaften kommunikativen Tun eine Konventionalisierung und Routinisierung erfahren, ob sich also bestimmte »Lösungen« der Indienstnahme von Emojis für einzelne kommunikative Aufgaben als Erfolgsmodelle im Gebrauch verfestigen und als solche in das überindividuelle, Situationen und Kontexte übergreifende Handlungsinventar vieler übergehen. Die Verwendergemeinschaft entscheidet im alltäglichen Handeln, welche FunktionsMittel-Zuordnungen sie für die Bearbeitung wiederkehrender kommunikativer Aufgaben benötigt, im geteilten Inventar festschreibt und tradiert. Wo sich Emojis heute (noch) als innovatives und originelles Mittel der Interaktionsgestaltung erweisen, mögen sie in wenigen Jahren feste Routine sein. Die von WhatsApp-Nutzenden immer wieder berichtete Erfahrung, dass im Bereich der privaten Alltagskommunikation die Nichtverwendung von Emojis häufig als intendierte Gestaltungsentscheidung ausgelegt würde (im Sinne von »Wer keine Emojis verwendet, verstößt bewusst gegen Normerwartungen und möchte damit etwas Bestimmtes ausdrücken«), kann als eine erste Tendenz in diese Richtung verstanden werden.

Funding Open Access funding provided by Projekt DEAL.

Open Access Dieser Artikel wird unter der Creative Commons Namensnennung 4.0 International Lizenz veröffentlicht, welche die Nutzung, Vervielfältigung, Bearbeitung, Verbreitung und Wiedergabe in jeglichem Medium und Format erlaubt, sofern Sie den/die ursprünglichen Autor(en) und die Quelle ordnungsgemäß nennen, einen Link zur Creative Commons Lizenz beifügen und angeben, ob Änderungen vorgenommen wurden. 
Die in diesem Artikel enthaltenen Bilder und sonstiges Drittmaterial unterliegen ebenfalls der genannten Creative Commons Lizenz, sofern sich aus der Abbildungslegende nichts anderes ergibt. Sofern das betreffende Material nicht unter der genannten Creative Commons Lizenz steht und die betreffende Handlung nicht nach gesetzlichen Vorschriften erlaubt ist, ist für die oben aufgeführten Weiterverwendungen des Materials die Einwilligung des jeweiligen Rechteinhabers einzuholen.

Weitere Details zur Lizenz entnehmen Sie bitte der Lizenzinformation auf http://creativecommons.org/ licenses/by/4.0/deed.de.

\section{Literatur}

Anderl, Sibylle (2017): Der richtige »Ton« im Netz. Der Mensch als Emoji. In: http://www.faz.net/aktuell/ wissen/die-macht-der-emojis-14672409.html (14.06.2017).

Auer, Peter (1999): Sprachliche Interaktion. Eine Einführung anhand von 22 Klassikern. Tübingen.

Beißwenger, Michael (2007): Sprachhandlungskoordination in der Chat-Kommunikation. Berlin/New York.

Beißwenger, Michael (2016a): Praktiken in der internetbasierten Kommunikation. In: Arnulf Deppermann/ Helmuth Feilke/Angelika Linke (Hg.): Sprachliche und kommunikative Praktiken. Jahrbuch 2015 des Instituts für Deutsche Sprache. Berlin/New York, S. 279-310.

Beißwenger, Michael (2016b): Sprache und Medien: Digitale Kommunikation. In: Studiport: Sprach- und Textverständnis. Hypermediales E-Learning-Angebot des Ministeriums für Innovation, Wissenschaft und Forschung (MIWF) des Landes Nordrhein-Westfalen. In: https://www.studiport.de/moodle/ course/index.php?categoryid=8, erweiterte, lineare Version: http://www.michael-beisswenger.de/ pub/beisswenger_digikomm_preview.pdf.

Beißwenger, Michael (2016c): Schriftliche Kommunikation im Netz. In: Ulf Abraham/Julia Knopf (Hg.): Deutsch digital. Band 2: Praxis. Baltmannsweiler. (Schriftenreihe Deutschdidaktik in der Primarstufe. Klassen 1-6), S. 58-67.

Beißwenger, Michael (2018): WhatsApp, Facebook, Instagram \& Co.: Schriftliche Kommunikation im Netz als Thema in der Sekundarstufe. In: Steffen Gailberger/Frauke Wietzke (Hg.): Deutschunterricht in einer digitalen Gesellschaft. Unterrichtsanregungen für die Sekundarstufen. Weinheim, S. 91-124.

Beißwenger, Michael (2020, i.Dr.): Internetbasierte Kommunikation als Textformen-basierte Interaktion: ein neuer Vorschlag zu einem alten Problem. In: Henning Lobin/Konstanze Marx/Axel Schmidt (Hg.): Deutsch in sozialen Medien: interaktiv, multimodal, vielfältig. Jahrbuch 2019 des LeibnizInstituts für Deutsche Sprache. Berlin/New York.

Beißwenger, Michael/Imo, Wolfgang/Fladrich, Marcel/Ziegler, Evelyn (2019): https://www.mocoda2.de: a database and web-based editing environment for collecting and refining a corpus of mobile messaging interactions. In: European Journal of Applied Linguistics (EuJAL) 7 (2), S. 333-344.

Beißwenger, Michael/Pappert, Steffen (2019a): Handeln mit Emojis. Grundriss einer Linguistik kleiner Bildzeichen in der WhatsApp-Kommunikation. Duisburg.

Beißwenger, Michael/Pappert, Steffen (2019b): Face work mit Emojis. Was linguistische Analysen zum Verständnis sprachlichen Handelns in digitalen Lernumgebungen beitragen können. In: Michael Beißwenger/Matthias Knopp (Hg.): Soziale Medien in Schule und Hochschule: Linguistische, sprach- und mediendidaktische Perspektiven. Frankfurt a. M., S. 99-141.

Beißwenger, Michael/Pappert, Steffen (2019c): How to be polite with emojis: a pragmatic analysis of face work strategies in an online learning environment. In: European Journal of Applied Linguistics (EuJAL), 7(2), S. 225-253.

Beißwenger, Michael/Storrer, Angelika (2012): Interaktionsorientiertes Schreiben und interaktive Lesespiele in der Chat-Kommunikation. In: Zeitschrift für Literaturwissenschaft und Linguistik 168, S. 92-124.

Cramer, Henriette/de Juan, Paloma/Tetreault, Joel (2016): Sender-Intended Functions of Emojis in US Messaging. In: Proceedings of the 18th International Conference on Human-Computer Interaction with Mobile Devices and Services. New York, NY: Association for Computing Machinery, 504-509. https://doi.org/10.1145/2935334.2935370 (26.11.2019).

Danesi, Marcel (2017): The Semiotics of Emoji. The Rise of Visual Language in the Age of the Internet. London/New York.

Deppermann, Arnulf/Feilke, Helmuth/Linke, Angelika (2016): Sprachliche und kommunikative Praktiken: Eine Annäherung aus linguistischer Sicht. In: Arnulf Deppermann/Helmuth Feilke/Angelika Linke 
(Hg.): Sprachliche und kommunikative Praktiken. Jahrbuch 2015 des Instituts für Deutsche Sprache. Berlin/New York, S. 1-23.

Dürscheid, Christa (2016): Neue Dialoge - alte Konzepte? Die schriftliche Kommunikation via Smartphone. In: Zeitschrift für germanistische Linguistik 44 (Themenheft Dialogizität), S. 437-468.

Dürscheid, Christa/Frick, Karina (2014): Keyboard-to-Screen-Kommunikation gestern und heute: SMS und WhatsApp im Vergleich. In: Alexa Mathias/Jens Runkehl/Torsten Siever (Hg.): Sprachen? Vielfalt! Sprache und Kommunikation in der Gesellschaft und den Medien. Eine Online-Festschrift zum Jubiläum für Peter Schlobinski (Networx 64), S. 149-181. In: https://www.mediensprache.net/de/ networx/networx-64.aspx (24.06.2019).

Dürscheid, Christa/Meletis, Dimitrios (2019): Emojis: A Grapholinguistic Approach. In: Yannis Haralambous (Hg.): Graphemics in the 21st Century. (Grapholinguistics and Its Applications 1). Brest, S. 167-183.

Dürscheid, Christa/Siever, Christina M. (2017): Jenseits des Alphabets - Kommunikation mit Emojis. In: Zeitschrift für germanistische Linguistik 45 (2), S. 256-285.

Ehlich, Konrad (1983): Text und sprachliches Handeln. Die Entstehung von Texten aus dem Bedürfnis nach Überlieferung. In: Aleida Assmann/Jan Assmann/Christof Hardmeier (Hg.): Schrift und Gedächtnis. Beiträge zur Archäologie der literarischen Kommunikation. (Archäologie der literarischen Kommunikation 1). München, S. 24-43.

Goffman, Erving (1977): Rahmen-Analyse. Ein Versuch über die Organisation von Alltagserfahrungen. Frankfurt a. M.

Gumperz, John J. (1982): Discourse Strategies. Cambridge.

Haase, Martin/Huber, Michael/Krumeich, Alexander/Rehm, Georg (1997): Internetkommunikation und Sprachwandel. In: Rüdiger Weingarten (Hg.): Sprachwandel durch Computer. Opladen, S. 51-85.

Heritage, John ( $\left.{ }^{3} 2010\right)$ : Conversation Analysis: Practices and methods. In: David Silverman (Hg.): Qualitative research. Theory, method and practice. London, S. 208-230.

Herring, Susan C./Dainas, Ashley R. (2017): »Nice picture comment!« Graphicons in Facebook comment threads. In: Proceedings of the Fiftieth Hawai'i International Con-ference on System Sciences (HICSS-50). Los Alamitos, CA. http://ella.slis.indiana.edu/ herring/hicss.graphicons.pdf (26.11.2019).

Imo, Wolfgang (2013): Sprache in Interaktion. Analysemethoden und Untersuchungsfelder. Berlin.

Imo, Wolfgang (2015): Vom Happen zum Häppchen ... Die Präferenz für inkrementelle Äußerungsproduktion in internetbasierten Messengerdiensten. In: Networx 69, S. 1-35. http://www.mediensprache.net/ de/networx/networx-69.aspx (26.11.2019).

Imo, Wolfgang (2017): Interaktionale Linguistik und die qualitative Erforschung computervermittelter Kommunikation. In: Michael Beißwenger (Hg.): Empirische Erforschung internetbasierter Kommunikation. Berlin/New York, S. 81-108.

Imo, Wolfgang (2019): Das Medium ist die Massage: Interaktion und ihre situativen und technischen Rahmenbedingungen. Erscheint in: Konstanze Marx/Axel Schmidt (Hg.): Interaktion und Medien. Heidelberg, S. 35-58.

Imo, Wolfgang/Fladrich, Marcel (2020, i.Dr.): Das Gelächter der Geschlechter 2.0. Emojigebrauch in der WhatsApp-Nutzung. In: Henning Lobin/Konstanze Marx/Axel Schmidt (Hg.): Deutsch in sozialen Medien: interaktiv, multimodal, vielfältig. Jahrbuch 2019 des Leibniz-Instituts für Deutsche Sprache. Berlin/New York.

Kelly, Ryan/Watts, Leon (2015): Characterising the inventive appropriation of emoji as relationally meaningful in mediated close personal relationships. Paper presented at Experiences of Technology Appropriation: Unanticipated Users, Usage, Circumstances, and Design, Oslo, Norway, 20 September. https://researchportal.bath.ac.uk/en/publications/characterising-the-inventive-appropriation-ofemoji-as-relational (26.11.2019).

Knoblauch, Hubert (1991): Kommunikation im Kontext: John. J. Gumperz und die Interaktionale Soziolinguistik. In: Zeitschrift für Soziologie, 20 (6), S. 446-462.

Kroeber-Riel, Werner (1996): Bildkommunikation. Imagerystrategien für die Werbung. München.

Pappert, Steffen (2017): Zu kommunikativen Funktionen von Emojis in der WhatsApp-Kommunikation. In: Michael Beißwenger (Hg.): Empirische Erforschung internetbasierter Kommunikation. Berlin/New York, S. 175-211.

Sachs-Hombach, Klaus (2003): Das Bild als kommunikatives Medium. Elemente einer allgemeinen Bildwissenschaft. Köln.

Schegloff, Emanuel (1997): Practices and actions: Boundary cases of other-initiated repair. In: Discourse Processes 23, S. 499-545.

Schmitz, Ulrich (2011): Sehflächenforschung. Eine Einführung. In: Hajo Diekmannshenke/Michael Klemm/Hartmut Stöckl (Hg.): Bildlinguistik. Theorien - Methoden - Fallbeispiele. Berlin, S. 23-42. 
Siebenhaar, Beat (2018): Funktionen von Emojis und Altersabhängigkeit ihres Gebrauchs in der WhatsApp-Kommunikation. In: Arne Ziegler (Hg.): Jugendsprachen. Aktuelle Perspektiven internationaler Forschung. Berlin/New York, S. 749-772.

Storrer, Angelika (2013): Sprachstil und Sprachvariation in sozialen Netzwerken. In: Barbara Frank-Job/ Alexander Mehler/Tilmann Sutter (Hg.): Die Dynamik sozialer und sprachlicher Netzwerke. Konzepte, Methoden und empirische Untersuchungen an Beispielen des WWW. Wiesbaden, S. 331-366.

Storrer, Angelika (2018): Interaktionsorientiertes Schreiben im Internet. In: Arnulf Deppermann/Silke Reineke (Hg.): Sprache im kommunikativen, interaktiven und kulturellen Kontext. Berlin/Boston, S. 219-244. https://doi.org/10.1515/9783110538601-010 